\title{
L'attachement préférentiel dans la formation d’alliances stratégiques
}

L'objectif de cet article est de mieux comprendre l'organisation des alliances stratégiques, ainsi que leur mode de fonctionnement dans le cas particulier des marchés publics. À partir de l'observation de 4203 alliances sur ces marchés, ce travail montre que la formation des coalitions d'entreprises se fait, en grande partie, selon un principe d'attachement préférentiel. Ce mécanisme de cohésion n'est pas sans conséquence sur la façon de gérer les alliances. Il implique que les structures relationnelles d'une entreprise déterminent le choix des partenaires. L'article présente finalement des éléments d'amélioration en matière d'organisation des alliances et propose différentes pistes de réflexions. 
$\mathrm{D}$ epuis la fin des années 1980, le nombre de cas et de formes d'alliances entre firmes s'est considérablement développé. Tous les secteurs économiques sont concernés, comme l'automobile, l'aéronautique, l'informatique, les télécommunications ou encore les banques. Il s'agit de secteurs, où les économies d'échelle et les effets d'expériences sont importants. Mais ces formes de rapprochements peuvent également concerner les industries dans lesquelles les dépenses de recherche et de développement (R\&D) sont élevées. Plus généralement, les activités à fortes intensités concurrentielles, qui nécessitent une présence ou des moyens élargis, incitent les entreprises à s'allier. Néanmoins, le recours aux alliances stratégiques s'accompagne d'un fort taux de « mortalité » (Meschi, 2006) en raison notamment de la difficulté à organiser et gérer ce type de relations dans la durée.

Fort de son importance, certains auteurs ont fait le choix de s'intéresser à l'organisation des alliances stratégiques (Gulati, 1999), en s'appuyant notamment sur plusieurs théories pour en comprendre le fonctionnement. On peut citer, à titre d'exemples, la théorie des coûts de transactions, l'approche des ressources et des compétences, la théorie de l'agence ou encore les travaux sur l'apprentissage organisationnel. Mais, si ces approches se complètent souvent, se contredisent parfois, aucun consensus, ni cadre intégrateur, ne permet de définir précisément la morphogenèse des alliances, c'est-à-dire leur formation, leur organisation et leur dynamique.

Or, pourtant, comme le montrent Makino et al. (2007), les conditions initiales de formation déterminent la survie et la performance d'une alliance stratégique. Il convient donc de mieux les comprendre pour en cerner les caractéristiques et leurs impacts sur les chances de réussite de futures alliances.

Notre question de recherche est donc la suivante : comment, en matière de formation d'alliances, une entreprise doit-elle sélectionner ses partenaires pour initier et former une coalition, dans le but d'obtenir des marchés, et d'assurer ainsi le développement de son organisation?

Pour y répondre, nous avons choisi d'étudier les alliances stratégiques au sein des marchés publics français à partir d'une approche structuraliste, compte tenu de l'importance des coalitions dans ce domaine. Le structuralisme est une approche des sciences humaines qui, dans le champ de la gestion, considère que les comportements stratégiques des acteurs peuvent s'expliquer par des mécanismes fondamentaux liés à un ensemble formel de structures de relations (Piaget, 1968). La nature des marchés étudiés incite à opter pour ce type de démarche (Voisin et al., 2004).

Aussi, l'objectif de cet article est de mieux cerner les conditions de formation des alliances et la sélection des entités partenaires, à partir de l'observation de 4203 alliances stratégiques, dans une perspective structurale (Borgatti et al., 2009). Ce travail débouche sur une modélisation du système d'interdépendance des acteurs au sein de l'environnement, permettant, à l'aide d'un graphe, d'apporter un éclairage nouveau sur les modes d'organisation des alliances stratégiques. Elle doit contribuer à mieux apprécier en quoi la formation des alliances se fait selon un principe d'attachement préférentiel, en vue de proposer des axes d'amélioration dans la gestion des relations de coopération. L'originalité de cet article se situe à deux niveaux. D'une 
part, il montre qu'au-delà des critères traditionnellement retenus dans la littérature (Geringer, 1988 ; Gulati et Gargiulo, 1999 ; Sorenson et Stuart, 2008), le principe d'attachement préférentiel est déterminant dans la sélection des partenaires d'une alliance. D'autre part, il met en exergue que le principe d'attachement préférentiel produit comme effet un avantage cumulatif (Merton, 1988) pour certaines entreprises. Ce résultat confirme que l'organisation des alliances repose sur une asymétrie relationnelle. Elle répond à un mécanisme inégalitaire qui est comparable à d'autres études empiriques, à l'instar des travaux sur la compétition académique (Carayol, 2006). Dans un premier temps, nous exposons les fondements théoriques des alliances stratégiques, ainsi que les apports de la théorie des graphes. Nous présentons, par la suite, la méthode utilisée pour collecter et étudier un grand nombre de relations d'alliances, en prenant les marchés publics français comme terrain d'étude. Enfin, nous discutons des résultats obtenus concernant l'attachement préférentiel, et présentons les implications managériales en matière de formation de coalitions.

\section{I - APPROCHES THÉORIQUES}

\section{Les alliances stratégiques}

L'analyse des alliances stratégiques constitue un champ de plus en plus important en sciences de gestion (Cheriet, 2007). Jolly (2001) définit l'alliance stratégique comme un lien entre plusieurs entreprises indépendantes qui partagent des ressources, afin de construire un avantage coopératif, dans le but d'atteindre des objectifs communs. La notion d'alliance stratégique induit, d'une part, une coopération, c'est-à-dire l'action de participer à une œuvre commune et, d'autre part, un engagement mutuel, c'est-à-dire une union qui a été négociée. Les alliances stratégiques se définissent ainsi comme des accords explicites, établis dans une perspective de plus ou moins longue durée. Au sein de ces formations, les entreprises partagent, combinent ou échangent des ressources, en vue de mener à bien un projet ou une activité spécifique. L'objectif de ces alliances est de bâtir et maintenir des avantages compétitifs plutôt que de réaliser ces activités de façon autonome (Philippart, 2001).

De manière générale, et malgré les controverses, un consensus se dégage dans la littérature sur les motivations des partenaires à former des alliances (Meier, 2009) : la minimisation des coûts de transactions, l'accès aux ressources et compétences, le transfert et l'apprentissage organisationnel, l'adaptation à l'environnement et à la structure compétitive d'un secteur. La littérature identifie également de nombreux critères pour sélectionner un partenaire (Cherni, 2010). Geringer (1988) distingue deux grandes catégories de critères de sélection : des critères liés aux ressources à partager (similitude ou complémentarité), et des critères liés aux comportements du partenaire (niveau d'engagement, compatibilité, contrôle de l'alliance). Pour une entreprise qui ne dispose pas des capacités ou des spécialités nécessaires à l'exécution d'un marché, la coopération constitue donc, un moyen privilégié pour obtenir des ressources. Au-delà des ressources qui contribuent directement à construire l'avantage concurrentiel, Geringer (1988) souligne que les actifs relationnels peuvent, également, être pris en compte. Par exemple, la connaissance du marché local, du contexte institutionnel, de même que les liens établis 
avec les acteurs locaux peuvent influencer le choix du partenaire. De plus, le niveau d'engagement dans l'accord d'alliances est, selon la littérature, un critère fondamental dans le choix du partenaire. L'engagement correspond à l'implication des partenaires pour fournir un effort, afin d'atteindre les objectifs communs. La confiance permettra d'appréhender le niveau d'engagement mutuel des partenaires dans la coopération et d'éviter les comportements opportunistes. Enfin, Geringer (1988) souligne l'importance de la compatibilité sur les plans stratégique et organisationnel dans la sélection des partenaires. La compatibilité des dirigeants, la culture d'entreprise et les procédures organisationnelles sont déterminantes dans la survie des alliances stratégiques.

\section{Les apports de la théorie des graphes}

Dans le cadre des alliances stratégiques, l'ensemble des relations interentreprises peut être considéré comme un réseau, c'està-dire « un système coordonné d'acteurs hétérogènes, développant des transactions fondées sur des relations de coopération, afin de poursuivre collectivement un objectif partagé » (Voisin et al., 2004). Le lien de coopération entre les entreprises se traduit dans la nature des échanges, leur périodicité, leur force, leur densité. Les entreprises partenaires occupent des positions, au sein du réseau d'alliances, qui sont susceptibles d'évoluer.

L'introduction de la théorie des graphes et de l'algèbre linéaire, dans l'étude des réseaux, a fait émerger, depuis les années 1970, un nouveau champ de recherche: la Social Network Analysis (SNA). Complété ensuite par des travaux issus des sciences physiques (Watts et Strogatz, 1998), ils vont permettre l'analyse de réseaux complexes. En effet, toutes les interactions d'un réseau peuvent être représentées par un graphe constitué d'un ensemble de points (les nœuds) reliés les uns aux autres par des liens (arcs).

L'analyse des grands réseaux révèle qu'ils possèdent tous un ensemble de propriétés mathématiques communes. En effet, les réseaux de connaissance (deux individus sont reliés s'ils se connaissent), les réseaux de contact physique (deux individus sont reliés s'ils ont été physiquement en contact), les réseaux de collaboration (deux individus sont reliés s'ils ont travaillé ensemble), les réseaux d'échanges (deux entités sont reliées si elles ont échangé un courrier électronique par exemple) sont tous organisés selon le principe d'attachement préférentiel.

\section{Le principe d'attachement préférentiel}

Issu des recherches sur les réseaux (Ahuja et al., 2009 ; Borgatti et al., 2009), le modèle d'attachement préférentiel a été développé par Barabàsi et Albert (1999) pour expliquer les phénomènes de regroupements. Il décrit comment des nœuds (individus, entreprises, etc.) se relient dans un graphe (réseau). Pour ces auteurs, dans un graphe dynamique, un nœud ne se connecte pas aux autres nœuds de façon aléatoire, mais avec une probabilité plus forte vers les nœuds les plus connectés. C'est le principe des « riches qui deviennent plus riches », c'est-à-dire, plus un nœud présente une connectivité importante par rapport aux autres, plus il attirera de nouveaux nœuds. Ce principe a des conséquences bien connues en économie, sous le nom d'effet Matthieu $^{1}$ (Carayol, 2006 ; Ederer, 2010). 
Par ce principe, les entreprises ayant déjà des partenaires ont un avantage cumulatif, car dans un "jeu dynamique ", les vainqueurs du tournoi de la première étape sont favorisés l'étape suivante du jeu.

La principale conséquence du principe d'attachement préférentiel est d'engendrer des "réseaux sans échelle» (scale free network). On parle de réseaux sans échelle car un calcul de grandeur moyenne n'a pas de sens. En effet, la distribution des connexions suit une loi de puissance dans laquelle peu de nœuds sont fortement connectés, tandis que beaucoup le sont faiblement. Ce principe remet en cause l'approche traditionnelle de l'attachement aléatoire connu jusqu'à présent en théorie des graphes, en montrant l'effet du temps (ancienneté) dans l'accroissement de la connectivité. L'une des explications de cet attachement préférentiel, dans les grands graphes de terrain, serait l'existence de "hubs» qui facilite la croissance du réseau (Barabàsi et Albert, 1999). Une autre explication de l'attachement préférentiel provient du fait que certains acteurs, pour réduire les incertitudes dans un marché, privilégient des relations avec des partenaires ayant un déjà statut élevé (Washington et Zajac, 2005). Comme

\section{MÉTHODOLOGIE}

Pour analyser les alliances stratégiques dans les marchés publics, nous avons retenu, comme méthode, l'approche structurale. Cette méthode permet de décrire les relations entre les entreprises, de façon simplifiée, à travers un graphe. Dans notre cas, l'analyse des alliances stratégiques repose sur les relations de coopération dans les groupements d'entreprises. Nous avons considéré qu'il y a une relation de coopération entre deux entreprises, lorsqu'elles obtiennent un marché dans le cadre d'une co-traitance. À partir des 54181 transactions réalisées au cours de l'année 2008, nous avons sélectionné, avec des mots clés, les transactions réalisées uniquement dans le cadre de groupements d'entreprises. Nous avons identifié 4203 transactions réalisées par des groupements, soit 10377 liens de coopération.

L'obtention de l'ensemble de ces données est un processus de plusieurs étapes de traitement : extraction, nettoyage, filtrage, formatage, dédoublonnage et indexation. La nature des données utilisées pour analyser les groupements d'entreprises, concerne trois variables. La première est un attribut lié à l'identification des acteurs (raison sociale). La deuxième concerne un attribut lié au type de bénéficiaires des transactions (groupements d'entreprises). La troisième variable concerne la relation de coopération qui relie les acteurs au sein d'un groupement.

Nous avons organisé les données relationnelles sous la forme d'une liste d'adjacence. La liste énumère, pour chaque acteur, tous les acteurs qui lui sont voisins. Avec la suite logicielle GraphViz, nous obtenons un graphe complet des relations de coopération au sein du marché public français en 2008 .

1. Selon l'évangile de saint Matthieu disant : «celui qui a, on lui donnera et il aura un surplus, mais celui qui n'a pas, même ce qu'il a lui sera enlevé ». 
le souligne Belvaux (2011), l'attachement préférentiel explique, par exemple, le développement du réseau collaboratif Wikipedia (Spinellis et Louridas, 2008), celui de Flickr (Mislove et al., 2008) ou plus généralement, des sites de commerce social (Stephen et Toubia, 2009).

\section{II - ANALYSE DES DONNÉES}

\section{Le choix des marchés publics}

Pour étudier l'organisation des alliances stratégiques, nous avons choisi les marchés publics français. Plusieurs raisons nous ont amenés à faire ce choix.

D'abord, la commande publique représente près de $10 \%$ du produit intérieur brut (PIB) de la France et renvoie à des enjeux économiques et politiques considérables. Le code des marchés publics définit un marché public comme un contrat conclu, à titre onéreux, entre un acheteur public (l'État, les ministères, les collectivités territoriales, les établissements publics comme les universités, lycée, certains musées...) et un opérateur économique public ou privé, pour répondre à ses besoins en matière de travaux, de fournitures ou de services. Un marché public est donc un acte juridique qui formalise la relation entre deux parties, et qui suppose la signature d'un contrat et le transfert d'un montant financier.

Ensuite, le principe de transparence dans les procédures relatives aux marchés publics oblige les donneurs d'ordre à faire la publicité des appels d'offres et des attributions des marchés. Cette transparence permet d'avoir, de façon exhaustive, les informations liées aux marchés publics. Les données utilisées pour cette recherche sont issues du fichier des avis d'attribution parus dans le Bulletin officiel des annonces des marchés publics (BOAMP) du Journal officiel $^{2}$. Le BOAMP publie les transactions attribuées par un donneur d'ordre public français, dont le montant du marché est supérieur à 4000 euros hors taxes.

Enfin, la réforme du code des marchés publics, initiée en 2006 et applicable depuis janvier 2008, encourage les réponses, par co-traitance, aux appels d'offres des marchés publics. La co-traitance illustre la situation dans laquelle des entreprises soumissionnaires peuvent s'allier dans un groupement d'entreprises, pour proposer une offre collective plus avantageuse que les offres individuelles. En fait, les groupements sont des systèmes où les agents tissent des liens pour échanger des biens ou des services, pour partager des étapes de création, de production ou de distribution, ou pour coordonner leurs activités. Il peut s'agir d'une coalition sous la forme d'un simple accord de partenariat, ce qui est le plus fréquent; mais également, une société en participation ou une société de fait, une société par action simplifiée, un groupement d'intérêt économique.

\section{La détection des réseaux d'alliances}

Il est proposé un rendu des différents résultats obtenus et de leurs conséquences sur la formation des alliances. Le graphe fait apparaître de nombreux agrégats dans lesquels des groupes de nœuds sont fortement liés entre eux, et faiblement liés à d'autres. En fait, ces agrégats sont des sous-graphes appelés composantes connexes, car il existe un chemin entre tout couple de nœuds. Le graphe est composé de 1360 composantes

2. Les avis d'attribution du BOAMP sont accessibles en ligne : www.journal-officiel.gouv.fr 
connexes, ayant entre 2 et 2233 nœuds. La connexion moyenne d'un nœud, dans le graphe, est de 3,16.

La figure 1 représente le graphe du système des relations interorganisationnelles des principales composantes connexes. Les entreprises sont symbolisées par des nœuds et leurs relations de coopération par des arcs. Le graphe permet d'identifier les règles dans la composition des alliances, et l'agencement des interdépendances entre les entreprises.

Le graphe des relations d'alliances stratégiques révèle un ensemble de propriétés topologiques non triviales :

- la structure du graphe fait apparaître des zones denses faiblement interconnectées, dans lesquelles des groupes d'entreprises sont fortement liés entre eux, et faiblement liés aux autres. Ces zones denses sont des composantes connexes et peuvent être définies comme des réseaux d'alliances,

- chaque entreprise est encastrée dans un réseau d'alliance,

- les entreprises ont des positions plus au moins centrales au sein de leur réseau d'alliance.

Le graphe de relations interentreprises engendre des structures de réseaux particulières. Ces réseaux d'alliances se construisent autour d'une entreprise « pivot» qui entretient des relations avec des entreprises partenaires. L'entreprisepivot est souvent le mandataire de la coa-

Figure 1 - Graphe des relations d'alliances stratégiques

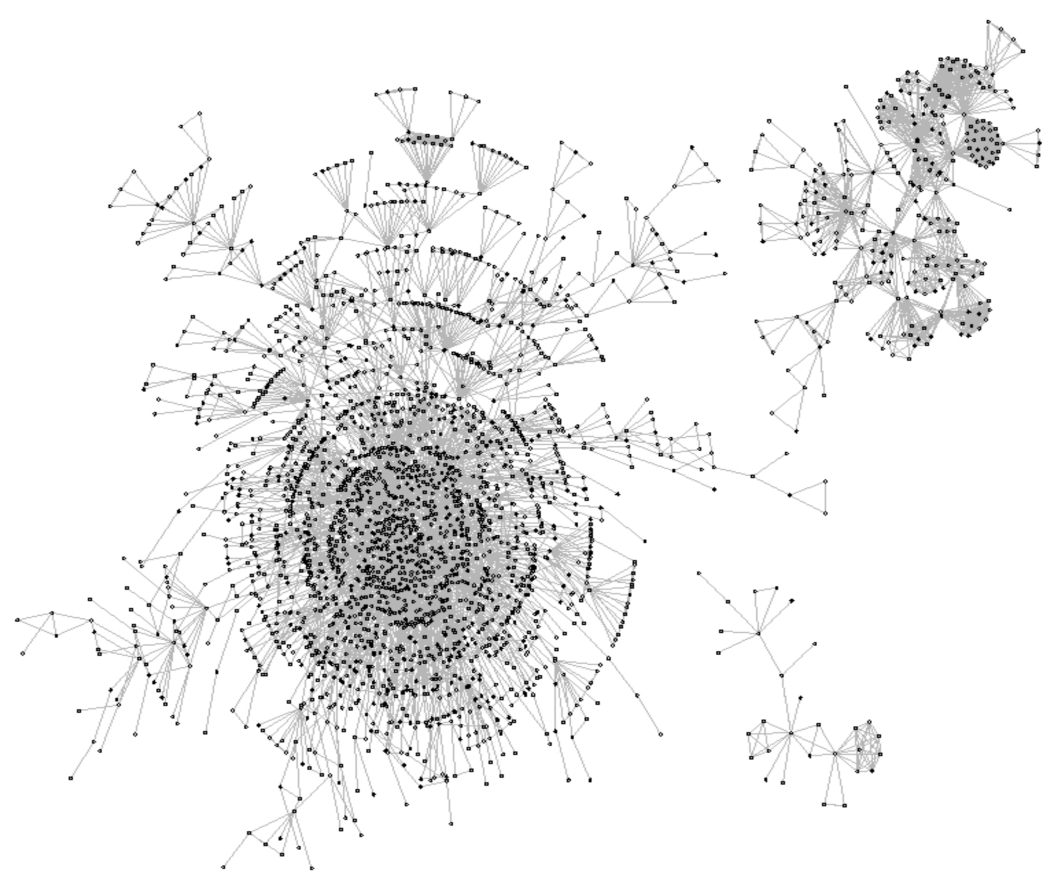


lition. Les entreprises partenaires de l'alliance gravitent autour de l'entreprise-pivot selon leurs structures relationnelles.

\section{L'organisation des alliances}

Pour mieux comprendre l'organisation des alliances dans les marchés publics, nous avons analysé la distribution de la centralité des degrés (figure 2). Cet indicateur correspond aux nombres de connexions qu'une entreprise a avec des partenaires dans le réseau. Il témoigne d'une position plus ou moins dominante dans le réseau. La distribution des connexions dans le réseau d'alliances est réalisée sous une échelle logarithmique. Ce type d'échelle est particulièrement adapté pour rendre compte des ordres de grandeur qui varient fortement. L'axe des ordonnées correspond aux nombres d'entreprises dans le réseau, tandis que l'abscisse correspond aux nombres de connexions.

La distribution montre que la majorité des entreprises ont une connexion faible, et quelques entreprises seulement ont une forte connexion. En fait, la distribution de la connectivité des entreprises est réglée par une loi de puissance dans laquelle près de $20 \%$ des entreprises attirent et génèrent $80 \%$ des liens du réseau. Ce type de distribution traduit l'évolution du réseau, car les nouvelles connexions se font principalement vers les entreprises qui en ont déjà beaucoup.

Figure 2 - Distribution des connexions dans le réseau d'alliances

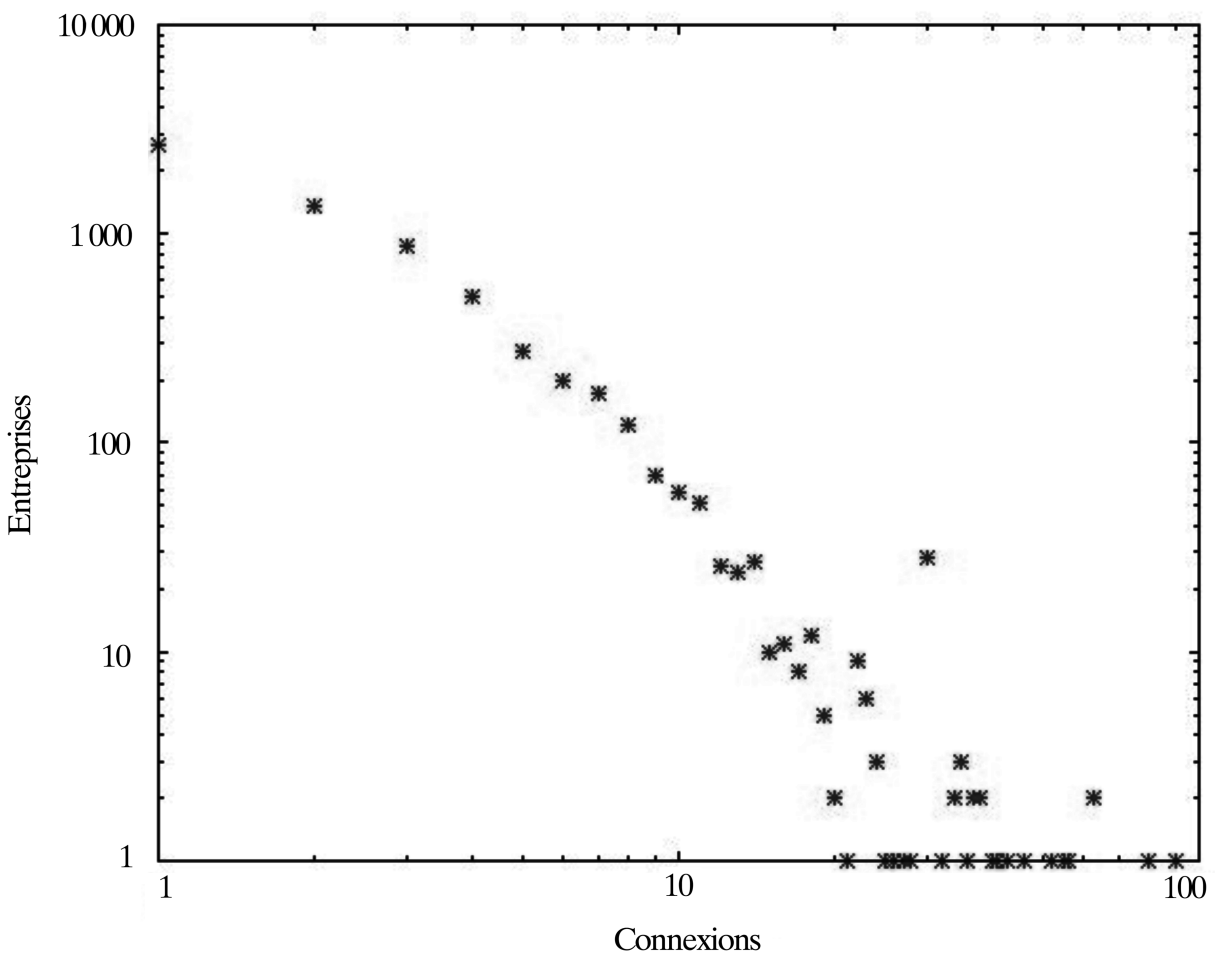


À partir de l'analyse statistique effectuée, il devient possible de considérer que la formation des alliances stratégiques se fait selon un principe d'attachement préférentiel. C'est-à-dire que la probabilité qu'une entreprise $A$ soit connectée à une entreprise $B$, dans le cadre d'une alliance, est proportionnelle au nombre de partenaires que l'entreprise $B$ possède déjà. En conséquence, plus une entreprise présente une connectivité importante, plus elle attirera des partenaires. Les résultats obtenus ont été trouvés, indépendamment des principaux critères retenus généralement dans la littérature sur la formation des alliances stratégiques.

\section{III - DISCUSSIONS}

L'analyse des résultats n'est pas sans conséquence dans la gestion stratégique des alliances. Notre étude montre tout d'abord que si les stratégies pour accéder aux marchés publics sont diverses, près de $8 \%$ des transactions de marchés publics sont obtenues par des alliances stratégiques. De plus, la recherche proposée nous conduit à approfondir plusieurs aspects en matière de formation d'alliances. De l'étude réalisée, il ressort, en particulier, trois points essentiels qu'il convient d'expliciter et de discuter, compte tenu de leur influence sur la formation des alliances et leur efficacité. Le premier point concerne le principe d'attachement préférentiel dans la sélection des partenaires. Notre étude souligne en effet l'importance de ce principe dans le choix des partenaires pour plusieurs raisons développées ci-après. Le deuxième apport concerne l'asymétrie de la position des partenaires et leurs effets sur le fonctionnement et le développement des réseaux d'alliances. Enfin, notre recherche insiste sur le rôle et l'intérêt d'une cartographie de l'environnement relationnel des entreprises pour limiter les risques et agir avec efficacité au sein des réseaux d'alliances.

La distribution des connexions dans le réseau d'alliances indique que les entreprises ayant déjà des partenaires sont plus attractives auprès des entreprises recherchant un nouveau partenaire. La raison est la suivante : choisir un partenaire qui a fait ses preuves, auparavant, permet d'augmenter les chances de l'entreprise dans une situation d'incertitude liée à l'attribution des marchés publics. Ce phénomène de choix consiste, en fait, à privilégier le partenaire auquel est associée la plus grande utilité espérée (Carayol, 2006). C'est-àdire que la sélection d'un partenaire est prise sur la base des performances passées, de ce dernier, en matière d'alliances. Cette motivation pour former une alliance s'explique par le fait que les entreprises soumissionnaires doivent avoir la position concurrentielle leur permettant de proposer l'offre la plus avantageuse. L'attribution d'un marché public se faisant généralement à partir d'un processus d'enchères, le choix d'une stratégie concurrentielle de soumission peut se résumer au dilemme suivant : soit accepter la rivalité, soit limiter l'intensité concurrentielle. Accepter la rivalité, c'est avant tout admettre que l'attribution du marché public se fera sur l'entreprise proposant le meilleur avantage concurrentiel. En général, cet avantage ne peut être obtenu qu'en proposant l'offre dotée du rapport qualité-prix le plus compétitif. En revanche, limiter la rivalité permet d'éviter la diminution du profit et augmente la probabilité d'obtenir un marché public dans un processus d'enchères. Dans ces conditions, 
l'alliance stratégique devient un moyen de limiter, voire d'éviter, la concurrence, dans la mesure où l'entreprise peut "négocier » avec ses concurrents de façon à anticiper les réactions de ceux-ci à ses propres décisions. La distribution des degrés montre que les partenaires, au sein d'une alliance, ont des positions stratégiques différentes. On repère ces entreprises statistiquement (figure 2), mais aussi visuellement à travers le graphe des relations d'alliances (figure 1). On peut ainsi noter que ces différences de positions créent une asymétrie relationnelle au sein des alliances stratégiques. Ce résultat traduit, ainsi, le rôle dévolu par certaines entreprises en matière de «hub» dans le fonctionnement et le développement des réseaux d'alliances. En fait, l'organisation des alliances favorise les partenaires qui ont déjà été les plus coopératifs. Car en absence d'information sur la capacité d'une entreprise à collaborer correctement dans le futur (c'est-à-dire la confiance à lui accorder), le nombre de partenaires passés constitue une garantie de fiabilité et un critère de sélection.

En conséquence, puisque la formation des alliances stratégiques se fait selon un modèle d'attachement préférentiel, on peut considérer que la sélection des partenaires est « hétérophile ». C'est-à-dire que les partenaires ont des niveaux hiérarchiques différents et sont motivés par la nécessité d'obtenir des ressources meilleures ou supplémentaires. Cette orientation est donc contraire à une sélection «homophile», dans laquelle les partenaires auraient la même position stratégique et dont la finalité serait de renforcer leur attractivité respective.

Enfin, l'étude nous renseigne sur l'importance de la gestion stratégique de l'en- vironnement. Elle montre que les entreprises qui voudront former des alliances stratégiques devront aussi développer leur capacité à analyser l'environnement, afin de détecter les réseaux d'alliances utiles, dans lesquels s'encastrer pour créer, et/ ou activer, des relations de coopération dans le cadre d'un projet défini. Mais ces stratégies relationnelles doivent souvent être proactives ou réactives, c'est-à-dire déterminées en réponse à la position effective ou supposée des concurrents. De plus, les manœuvres stratégiques variant dans le temps, en fonction des opportunités et des parties prenantes, à l'instar de ce que suggère Baumard (2000), tout l'enjeu consiste à comprendre les mouvements stratégiques des concurrents, pour faire de leurs asymétries, le levier de la construction de l'avantage concurrentiel. Ainsi, face à un environnement dynamique et instable, l'apport d'une cartographie peut constituer une aide précieuse qui permettra la réduction de la part d'incertitude dans la prise de toute décision stratégique. Les intérêts d'une cartographie sont en effet multiples : représenter les entreprises et leurs relations, naviguer dans les réseaux d'entreprises, identifier la position de chaque entreprise sur le marché, mesurer la force des liens (affinités) entre les entreprises.

\section{CONCLUSION}

Nous avons mené une étude sur 4203 alliances stratégiques qui ont obtenu des marchés publics en 2008. Cette étude nous a permis, à partir d'une analyse structurale, de mettre à jour les réseaux dans lesquels sont encastrées les alliances stratégiques. Elle révèle, ensuite, que la formation des alliances stratégiques, dans les 
marchés publics, se fait selon un principe d'attachement préférentiel. L'attachement préférentiel se traduit par la distribution des connexions au sein des réseaux. Cette distribution, en loi de puissance, est très caractéristique des grands réseaux, appelés : réseau sans échelle (Barabàsi et Albert, 1999). L'émergence de ce type de réseau est déterminée par des acteurs capables d'envoyer un signal pour attirer de nouveaux liens et par leur capacité à exploiter un avantage cumulatif. Cet avantage cumulatif crée une dynamique vertueuse qui favorise la création de nouvelles alliances dans l'accès aux marchés publics français. Sur le plan théorique, cet article montre, d'une part, que les propriétés structurales, mises à jour dans les recherches issues des réseaux sociaux, sont également valides dans les réseaux interorganisationnels. Il complète ainsi les travaux initiés par Gulati (1999) sur les « réseaux stratégiques». D'autre part, cet article confirme que les alliances stratégiques sont basées, en grande partie, sur des relations asymétriques entre les partenaires (Assens et Cherbib, 2010). Il suggère notamment la centralité de degré dans les réseaux comme une nouvelle variable d'asymétrie.

D'un point de vue managérial, cette recherche permet, de plus, de formuler des recommandations aux entreprises, dans le but d'accéder aux marchés publics à partir d'une stratégie de coopération. En effet, les structures relationnelles d'une entreprise déterminent le choix de ses partenaires. En conséquence, plus une entreprise a de partenaires, plus elle a intérêt à consolider son pouvoir de négociation et à assurer un rôle de leader, en sélectionnant des partenaires peu influents. De même, moins une entreprise dispose de partenaires et plus elle a intérêt à s'associer avec un partenaire qui domine le réseau. Tout l'enjeu, alors, est de pouvoir développer des capacités d'apprentissage pour acquérir les connaissances et les ressources du partenaire dominant.

À partir de ce modèle d'organisation des coalitions, plusieurs perspectives sont envisageables. Trois pistes nous semblent particulièrement intéressantes à développer. Tout d'abord, identifier les autres propriétés structurales qui expliquent la formation des alliances stratégiques (Mandard, 2012). Par exemple, quelle est la probabilité que deux entreprises réalisent un partenariat si elles possèdent des relations communes avec d'autres entreprises ?, ou bien encore, quelle est l'influence de liens répétés entre deux acteurs dans la création de nouveaux partenariats? De plus, une étude longitudinale permettrait de vérifier l'effet des structures relationnelles sur la performance des alliances stratégiques, notamment dans l'attribution de marchés publics. Enfin, le recours à une analyse structurale, dans le cadre de recherches ultérieures, serait d'un grand apport pour comprendre l'instabilité et la survie des alliances. 


\section{BIBLIOGRAPHIE}

Ahuja G., Soda G., Zaheer A. (2009). "The genesis and dynamics of organizational networks", Organization Science, June, p. 1-44.

Assens C., Cherbib J. (2010). «L'alliance asymétrique : une stratégie durable ? », La Revue des Sciences de Gestion, vol. 3, n²43-244, p. 111-119.

Barabàsi A.L., Albert R. (1999). "Emergence of scaling in random networks", Science, vol. 286, n 5439 , p. 509-512.

Baumard P. (2000). Analyse stratégique: mouvements, signaux concurrentiels et interdépendance, Dunod, Paris

Belvaux B. (2011), « Le développement des médias sociaux. Proposition d'un modèle de diffusion intégrant les externalités de réseau dans un cadre concurrentiel », Recherche et Applications en Marketing, vol. 26, n 3, p. 7-22.

Borgatti S.P., Mehra A., Brass D.J., Labianca G. (2009). "Network analysis in the social sciences", Science, New York, N.Y., vol. 323, n 5916, p. 892-895.

Carayol N., (2006), « Les propriétés incitatives de l'effet saint matthieu dans la compétition académique », Revue économique, vol. 57, n 5, p. 1033-1051.

Cheriet F. (2007). «Alliances stratégiques et relations interentreprises dans la recherche francophone en stratégie : Bilan des contributions de l'AIMS entre 1996 et 2006 », AIMS, Montréal.

Cherni M. (2010). Les critères de sélection de partenaires dans les coopérations d'innovation, thèse, École doctorale Sciences de Gestion.

Gulati R., Gargiulo M. (1999). "Where do interorganizational networks come from?”, American Journal of Sociology, vol. 104, n 5, p. 1439-1493.

Ederer F. (2010). "Feedback and motivation in dynamic tournaments", Journal of Economics and Management Strategy, 19, p. 733-769.

Geringer J.M. (1988). Joint venture partner selection, West Port, Quorum Books.

Gulati R. (1999). "Network location and learning: The influence of network resources and firm capabilities on alliance formation", Strategic Management Journal, vol. 20, $\mathrm{n}^{\circ} 5$, p. 397-420.

Jolly D. (2001). Alliances interentreprises (les), Vuibert, p. 163.

Makino S., Chan C.M., Isobe T., Beamish P.W., (2007). "Intended and unintended termination of international joint ventures", Strategic Management Journal, vol. 28, $\mathrm{n}^{\circ} 11$, p. 1113-1132.

Mandard, M. (2012). «L'influence des réseaux interorganisationnels sur les partenariats d'entreprises », Management \& Avenir, 51, p. 99-115.

Meier O. (2009). Stratégies de croissance, Paris, Dunod. 
Merton, R.K. (1988). "The Matthew effect in science, II: Cumulative advantage and the symbolism of intellectual property", Isis, vol. 79, p. 606-623

Mislove A., Koppula H.S., Gummadi K.P., Druschel P. et Bhattacharjee B. (2008). "Growth of the Flickr social network", Workshop on Online Social Networks, Seattle.

Meschi P.-X. (2006). "Réseaux interorganisationnels et survie des alliances », Revue française de gestion, vol. 32, $\mathrm{n}^{\circ} 164$, p. 33-54.

Philippart P. (2001). « La gestion des alliances interentreprises : Précision des enjeux à partir d'une revue de littérature ordonnée en trois pôles de problématiques », $X^{e}$ conférence de l'AIMS, Québec, 13-15 juin.

Piaget J. (1968). Le structuralisme, Paris, PUF.

Sorenson O., Stuart T.-E. (2008). "Bringing the context back in: Settings and the search for syndicate partners in venture capital investment networks", Administrative Science Quarterly, vol. 53, n², p. 266-294.

Spinellis D. et Louridas P. (2008). "The collaborative organization of knowledge", Communications of the ACM, vol. 51, $\mathrm{n}^{\circ} 8, \mathrm{p} .68-73$.

Stafford, E. (1994). "Using Co-operative Strategies to make alliances work", Long Range Planning, vol. 27, $\mathrm{n}^{\circ}$ 3, p. 64-74.

Stephen A.T. et Toubia O. (2009). "Explaining the power law degree distribution in a social commerce network", Social Networks, vol. 31, n 4, p. 262-270.

Voisin C., Mahmoud-Jouini S., Ben Édouard S. (2004). Les réseaux: dimensions organisationnelles et stratégiques, Economica.

Washington M., Zajac E.J. (2005). "Status evolution and competition: theory and evidence", Academy of Management Journal, vol. 48, n 2, p. 282-296.

Watts D.J., Strogatz S.H. (1998). “Collective dynamics of small-world networks", Nature, vol. $393, n^{\circ} 6684$, p. $440-442$. 
